\title{
Full Counting Statistics of Electron Transfer between Superconductors
}

\author{
W. Belzig* and Yu. V. Nazarov \\ Department of Applied Physics and Delft Institute of Microelectronics and Submicrontechnology, \\ Delft University of Technology, Lorentzweg 1, 2628 CJ Delft, The Netherlands
}

(Received 7 December 2000; published 23 October 2001)

\begin{abstract}
We present an extension of the Keldysh-Green's function method, which allows one to calculate the full distribution of transmitted particles through a mesoscopic superconductor. The method is applied to the statistics of supercurrent in short contacts. If the current is carried by Andreev bound states the distribution corresponds to switching between long trains of electrons going in opposite directions. For weak (gapless) superconductors or tunnel junctions we find that at low temperatures the distribution has negative "probabilities." Accounting for the quantum mechanical nature of the measuring device shows that these negative values can indeed be measured.
\end{abstract}

DOI: 10.1103/PhysRevLett.87.197006

Coherent charge transfer between superconductors (S), supercurrent, is essentially a quantum mechanical process. Although superconducting junctions are commonly used, the statistical properties of the charge transfer involved in the supercurrent are not yet completely understood. In view of recent attempts to use the coherence of superconductors to build quantum bits [1], it is necessary to reveal the basic limitations on this coherence (if there are any). Additionally, the problem of the statistics of transferred charge in a quantum process is of fundamental interest. It is related to the understanding of the measurement process and the interpretation of its outcome.

Recently the current noise exhibited in SNS junctions, where $N$ is a diffusive normal metal, was addressed experimentally [2]. The experimental results show a giant excess noise in the low temperature and voltage regime in those samples, in which at the same temperature a coherent coupling through the normal metal was measured. This is in accordance with theoretical predictions for the shot noise in short contacts [3]. This may hint to the importance of an understanding of the statistical properties of the supercurrent in such junctions. The equilibrium noise properties have been studied in [4] and [5]. Further experimental progress in the fabrication of controllable single-channel junctions is to be expected in the near future. This will shed more light on the fundamental statistical properties of charge transfer between superconductors.

We will make use of the so-called full counting statistics (FCS), originally introduced to calculate the distribution of transmitted charge through a contact between normal metals [6]. This theory allows one to find the cumulant generating function (CGF) $S(\chi)$, from which the distribution of transmitted charge follows via $P(N)=$ $\int d \chi \exp [-S(\chi)-i N \chi]$. It is tempting (and has been done so far) to interpret $P(N)$ as the probability that $N$ charges have been transferred through the contact during the time of observation. We will show below that this interpretation is strict only for normal constrictions. For superconducting constrictions the distribution also depends on the phase difference $\phi$. It turns out that $P(N, \phi)$ can
PACS numbers: 74.50.+r, 05.40.-a, 72.70.+m, 73.23.-b

also take negative values, which hampers such interpretation. This is related to the fact that the phase and number of charges transferred can be regarded as canonically conjugated variables. Still $P(N, \phi)$ provides a complete description of all charge transfer processes and can be extracted from the results of measurements.

The most powerful and general method of calculating transport properties of mesoscopic conductors is the nonequilibrium Green's function approach (see [7]). It was shown in [8] that this approach can be generalized to access FCS. In this Letter we extend the approach to superconductors. This allows us to obtain the FCS of an arbitrary mesoscopic conductor at all temperatures and voltages. The CGF is derived for a contact which is fully characterized by an ensemble of transmission eigenvalues $\left\{T_{n}\right\}$. We evaluate the FCS of supercurrent in two generic cases. First, we find the distribution of transmitted charge of a single-channel contact between two gapped superconductors. Here the current is carried by phase dependent Andreev bound states and, as shown by our analysis, conforms with the switching picture [5]. The two bound states carrying current in opposite directions are alternately occupied and charges are transferred in "long trains," which reflect the coherent nature of the supercurrent. In the second case we calculate the CGF of a contact between two weak superconductors. The resulting CGF corresponds to the tunnel limit for gapped superconductors and can be related to the effective Keldysh action of a Josephson junction discussed in detail in Ref. [9]. The standard interpretation [6] of the CGF leads in the low temperature regime to negative "probabilities" $P(N, \phi)$. Negative values of $P(N, \phi)$ occur because of an attempt to interpret the quantum mechanical phenomenon of supercurrent with classical means. If we account for the quantum mechanical nature of the measuring device, we can resolve the paradox and specify how $P(N, \phi)$ can be measured.

To be specific, let us now introduce our model system, which is depicted in Fig. 1. A mesoscopic conductor is placed between two reservoirs. The counting field $\chi$ is introduced on an arbitrary cross section in one of the 


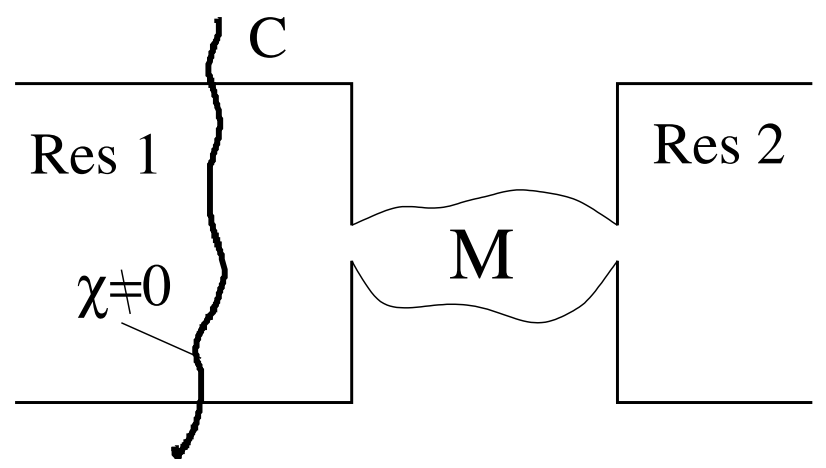

FIG. 1. Sketch of the system. Two reservoirs $(1,2)$ are connected to a mesoscopic conductor $\mathrm{M}$. The counting field $\chi$ is chosen nonzero on the cross section $\mathrm{C}$ in reservoir 1 .

reservoirs and couples to the operator of current through that cross section. It follows from the definition of the $\mathrm{cu}-$ mulants that the CGF can be found from

$$
e^{-S(\chi, \phi)}=\left\langle\mathcal{T} e^{i(\chi / 2) \int_{0}^{t 0} \hat{I}(t) d t} \tilde{\mathcal{T}} e^{i(\chi / 2) \int_{0}^{t 0} \hat{I}(t) d t}\right\rangle .
$$

Here $\mathcal{T}(\tilde{\mathcal{T}})$ denotes the (anti)time ordering operator. $\hat{I}$ denotes the current operator $\int d^{3} x \hat{\Psi}^{\dagger} \bar{\tau}_{3}(\boldsymbol{p} / m) \hat{\Psi}^{\dagger} \nabla F(\boldsymbol{x})$, where $\hat{\Psi}$ is the usual Nambu spinor field operator and $\bar{\tau}_{3}$ is a matrix in Nambu space. $\nabla F$ is chosen such that the spatial integration is restricted to the cross section and yields the total current. The counting field parametrized in this way can now be incorporated into the boundary condition imposed by the reservoir onto the mesoscopic conductor [8]. That is, the reservoir Green's function effectively takes the form

$$
\check{G}_{1}(\chi, \phi)=e^{(i / 2) \chi \check{\tau}_{\mathrm{K}}} \check{G}_{1}(\phi) e^{-(i / 2) \chi \check{\tau}_{\mathrm{K}}} .
$$

Here $\check{G}_{1}(\phi)$ is the reservoir Green's function at superconducting phase $\phi$ in the absence of the counting field and $\check{\tau}_{\mathrm{K}}=\hat{\sigma}_{3} \bar{\tau}_{3}$ a matrix in Keldysh(^)-Nambu(-) space. Now the counting field is included in the boundary condition for the Keldysh-Nambu matrix Green's functions provided by the left reservoir. Inside the system of interest the transport properties are described by quasiclassical Eilenberger equations [10], applicable if the system size exceeds the Fermi wavelength. It is important that $\breve{G}(\chi, \phi)$ still obeys the quasiclassical normalization condition $\breve{G}^{2}(\chi, \phi)=1$.

In certain cases the action $S(\chi, \phi)$ can be found quite generally. One example is a constriction shorter than the coherence length, which is fully characterized by a set of transmission eigenvalues $\left\{T_{n}\right\}$. The counting field manipulates the matrix structure of the Green's functions in Keldysh-Nambu space. To find the transport properties one should therefore use expressions, which respect the full matrix structure. It was noted in [11] that a convenient way to do this is to use a "matrix current," which is conserved in short contacts. The matrix current is formed with the current operator and the corresponding matrix elements of the Green's functions. Physical currents are related to certain components of the matrix current. For our purpose here, we need the matrix current in a short contact.
The matrix current was derived in [11] in the absence of the counting field. The counting field does not change the matrix structure of that result. So we can use it just by including the $\chi$ dependence of the Green's functions and write

$$
\check{I}(\chi, \phi)=\frac{1}{2 \pi} \sum_{n} \int d E \frac{T_{n}\left[\check{G}_{1}(\chi, \phi), \check{G}_{2}\right]}{4+T_{n}\left(\left\{\check{G}_{1}(\chi, \phi), \check{G}_{2}\right\}-2\right)} .
$$

The action can then be found from the relation $(\partial / \partial \chi) \times$ $S(\chi, \phi)=-i t_{0} \operatorname{Tr}\left(\check{\tau}_{\mathrm{K}} \check{I}(\chi, \phi)\right)$. Using the fact that $\left[\breve{A},\left\{\breve{A}, \breve{G}_{2}\right\}\right]=0$ for all matrices with $\breve{A}^{2}=\breve{1}$, it is easy to verify that under the trace in (3) $(\partial / \partial \chi)\left\{\check{G}_{1}(\chi, \phi), \breve{G}_{2}\right\}=$ $i \check{\tau}_{K}\left[\breve{G}_{1}(\chi, \phi), \breve{G}_{2}\right]$. We can therefore integrate Eq. (3) with respect to $\chi$ and obtain

$$
\begin{aligned}
S(\chi, \phi)= & \frac{-t_{0}}{2 \pi} \sum_{n} \int d E \\
& \times \operatorname{Tr} \ln \left[4+T_{n}\left(\left\{\check{G}_{1}(\chi, \phi), \check{G}_{2}\right\}-2\right)\right] .
\end{aligned}
$$

Equation (4) is very general. It contains the statistical properties of all types of superconducting constrictions. For instance, the FCS of an SN contact [12] can easily be obtained from (4).

In the rest of the paper we will study equilibrium noise and statistics of systems with two superconducting contacts. We will distinguish two generic cases. The first will be a single-mode contact of arbitrary transparency between two fully gapped superconductors. In the second case we treat a contact between two weak superconductors, or, which is equivalent, a tunnel contact. The channel summation in the action (4) is then a trivial summation over transparencies. In the following derivation we limit the discussion to a single channel of transmission $T_{1}$ and identical reservoirs at equilibrium. To be specific, we consider the Green's functions of the reservoirs

$$
\check{G}_{S}=\frac{\bar{R}+\bar{A}}{2}+\frac{\bar{A}-\bar{R}}{2}\left(\begin{array}{cc}
-h & (1-h) \\
(1+h) & h
\end{array}\right) .
$$

Here $\bar{R}(\bar{A})(E)$ are retarded and advanced Green's functions of the banks and $h(E)=\tanh (E / 2 T)$ accounts for the equilibrium distribution at a temperature $T$. The phase difference $\phi$ is introduced by setting $\breve{G}_{1}(\phi)=$ $\exp \left(i \phi \bar{\tau}_{3} / 2\right) \check{G}_{S} \exp \left(-i \phi \bar{\tau}_{3} / 2\right)$ and $\breve{G}_{2}=\check{G}_{S}$. Advanced and retarded functions in (5) possess the structure $\bar{R}(\bar{A})=g_{\mathrm{R}, \mathrm{A}} \bar{\tau}_{3}+f_{\mathrm{R}, \mathrm{A}} \bar{\tau}_{1}$ fulfilling the normalization condition $f^{2}+g^{2}=1$. They depend on energy and the superconducting order parameter $\Delta$. Their precise forms will be defined below.

The trace in the action can be evaluated, and we obtain the main result of this paper

$S(\chi, \phi)=\frac{-t_{0}}{\pi} \int d E \ln \left[1+\sum_{n=-2}^{2} \frac{A_{n}(\phi)}{Q(\phi)}\left(e^{i n \chi}-1\right)\right]$.

Introducing $q=\left(1-g_{\mathrm{R}} g_{\mathrm{A}}\right)\left(1-h^{2}\right)+f_{\mathrm{R}} f_{\mathrm{A}}\left(1+h^{2}\right)$ 
the coefficients may be written as

$$
\begin{gathered}
A_{ \pm 2}=\frac{T_{1}^{2}}{64} q^{2} \\
A_{ \pm 1}=\frac{T_{1}}{4} q-\frac{T_{1}^{2}}{16} q\left[q-4 f_{\mathrm{R}} f_{\mathrm{A}} \sin ^{2} \frac{\phi}{2}\right] \\
+\frac{T_{1}}{8}\left[\left(f_{\mathrm{R}}+f_{\mathrm{A}}\right) h \cos \frac{\phi}{2}\right. \\
\left.\mp i\left(f_{R}-f_{\mathrm{A}}\right) \sin \frac{\phi}{2}\right]^{2} \\
Q=\left[1-T_{1} f_{\mathrm{R}}^{2} \sin ^{2}\left(\frac{\phi}{2}\right)\right]\left[1-T_{1} f_{\mathrm{A}}^{2} \sin ^{2}\left(\frac{\phi}{2}\right)\right] .
\end{gathered}
$$

The interpretation of the different terms is analogous to that given in [12]. A coefficient $A_{ \pm n}$ is related to events in which a charge $n$ is transferred to the right (left). The presence of terms, which describe charge transfers of $2 e$, is a consequence of superconducting correlations. The interpretation of these terms as probabilities stems from the comparison with the case of binomial statistics (see [6]). However, in our case $Q$ has roots for the Andreev bound state energies. The coefficients $A_{ \pm n} / Q$ can become larger than 1, inhibiting an interpretation as probabilities. To find the statistics of the charge transfer, we have to specify the system further.

Gapped superconductors. - If the two leads are gapped like BCS superconductors, the spectral function are given by $f_{\mathrm{R}, \mathrm{A}}=i \Delta /\left[(E \pm i \delta)^{2}-\Delta^{2}\right]^{1 / 2}$ and $g_{\mathrm{R}, \mathrm{A}}$ follows from normalization. Here $\delta$ is a broadening parameter, which accounts for the finite lifetime of the Andreev bound states due to, e.g., phonon scattering. The supercurrent is solely carried by Andreev bound states with energies $\pm \Delta\left(1-T_{1} \sin ^{2} \phi / 2\right)^{1 / 2} \equiv \pm E_{\mathrm{B}}(\phi)$. The importance of these bound states can be seen from the coefficient $Q$ (9). It may become zero and will thus produce singularities in the action [13]. The broadening $\delta$ shifts the singularities of $Q$ into the complex plane and allows an expansion of the coefficients in Eqs. (7)-(9) close to that energy. Performing the energy integration the action results in

$$
S(\chi, \phi)=-2 t_{0} \delta \sqrt{1-I_{1}^{2}(\phi) \chi^{2} / 4 \delta^{2}-i \chi \bar{I}_{1}(\phi) / \delta},
$$

where $I_{1}(\phi)=\Delta^{2} T_{1} \sin (\phi) / 2 E_{\mathrm{B}}(\phi)$ is the supercurrent carried by one bound state and $\bar{I}_{1}(\phi)=I_{1}(\phi) \times$ $\tanh \left(E_{\mathrm{B}} / 2 T\right)$ is the average current through the contact. In deriving (10) we have also assumed that $\chi \ll 1$. This corresponds to a restriction to "long trains" of electrons transferred, and the discreteness of the electron transfer plays no role here. Fast switching events become less probable at low temperatures and are neglected here. In the saddle point approximation at low temperatures $\gamma \equiv 1 /$ $\cosh \left(E_{B} / 2 T\right) \ll 1$ we find for the current distribution

$$
P(j, \phi) \sim \frac{1}{\gamma} e^{2 \delta t_{0}\left(\gamma \sqrt{1-j^{2}(\phi)}-j(\phi) \sqrt{1-\gamma^{2}}\right)},
$$

for $|j(\phi)| \leq 1$ and zero otherwise. Here we have expressed the transferred charge in terms of the current normalized to the zero temperature supercurrent: $j(\phi)=$ $I / I_{1}(\phi)$. The current is related to the particle number by $N=I t_{0}$. At zero temperature Eq. (11) approaches $P(j, \phi) \rightarrow \delta(j-1)$, which follows from a direct calculation. Thus, at zero temperature the current is noiseless and the distribution (11) at finite temperature confirms the picture of switching between Andreev states which carry current in opposite directions, suggested in Ref. [5]. These results are valid, as long as the bound states are well separated from each other and from the continuum. Otherwise the statistics are similar to the gapless case.

Tunnel junction/gapless superconductors. - Let us now consider the supercurrent statistics between two weak superconductors, where the Green's functions can be expanded in $\Delta$ for all energies. One can see that this is equivalent to the tunneling limit $\left(\left\{T_{n}\right\} \ll 1\right)$ of Eq. (4). We also return the many channel situation here. Expanding the action (4) to lowest order and using that the counting rotation can be written as $\exp \left(i \chi \check{\tau}_{K} / 2\right)=\frac{1}{2}\left[e^{i \chi / 2}\left(1-\check{\tau}_{K}\right)+\right.$ $\left.e^{-i \chi / 2}\left(1+\check{\tau}_{K}\right)\right]$ we find

$$
S=-t_{0}\left[i I_{\mathrm{s}}(\phi) \sin \chi+P_{\mathrm{s}}(\phi)(\cos \chi-1)\right] .
$$

In short, the full statistics are expressed in terms of supercurrent $I_{\mathrm{s}}(\phi)$ and noise $P_{\mathrm{s}}(\phi)$. In equilibrium using (5)

$$
\begin{gathered}
I_{\mathrm{s}}(\phi)=-\frac{G}{4} \operatorname{Re} \int d E \operatorname{Tr}\left\{\bar{\tau}_{3}\left[\bar{R}_{1}(\phi), \bar{R}_{2}\right]\right\} h, \\
P_{\mathrm{s}}(\phi)=-\frac{G}{4} \operatorname{Re} \int d E \operatorname{Tr}\left\{\bar{\tau}_{3} \bar{A}_{1}(\phi) \bar{\tau}_{3} \bar{R}_{2}\right\}\left(1-h^{2}\right) .
\end{gathered}
$$

Here $G=(1 / \pi) \sum T_{n}$ is the normal state conductance of the contact. The equivalence of this result to the limit of gapless superconductors follows from an expansion of (4) -(9) to orders $f^{2}$.

Equation (14) shows that $P_{\mathrm{s}}$ vanishes at zero temperature, since $h(T=0)= \pm 1$, whereas $I_{\mathrm{s}}$ vanishes at $T_{\mathrm{c}}$. Therefore, there is some crossover temperature below which $P_{\mathrm{s}}<I_{\mathrm{s}}$. In this limit the action possesses no saddle point anymore, and by expansion in powers of $\exp (i \chi)$ it follows that $P(N, \phi)$ becomes negative. Obviously this questions the direct interpretation of $P(N, \phi)$ as a probability. Thus, we are forced to have a closer look on what $P(N, \phi)$ actually is.

To clarify this issue, we make use of the recent results presented in [14], where it was shown that the interpretation of $P(N, \phi)$ is intimately related to the way the measurement is performed. We assume a simple model of a measuring device: a capacitor of infinite capacitance that stores the charge passed through the constriction; i.e., the charge operator $\hat{q}$ is related to the current operator through 
the constriction by $\dot{\hat{q}}=\hat{I}$. The quantum mechanical treatment of this device involves its density matrix $\rho\left(q, q^{\prime}\right)$.

In [14] the relation between initial and final density matrices of the device was obtained. This can be expressed in terms of the density matrix in Wigner representation, $\rho(x, q), x$ being the canonical conjugate of $q$. It reads

$$
\rho_{f}(x, q)=\sum_{N} P(N, \phi-x) \rho_{i}(x, q-N),
$$

so that $P(N, \phi)$ fully characterizes the quantum mechanical behavior of the capacitor. For a normal constriction $P(N, \phi)$ does not depend on $\phi$. In this case we can rewrite Eq. (15) directly in terms of charge distributions $\Pi(q) \equiv$ $\int d x \rho(x, p)$,

$$
\Pi_{f}(q)=\sum_{N} P(N) \Pi_{i}(q-N) .
$$

Therefore, $P(N)$ can be interpreted as classical probability. For a superconducting constriction quantum mechanics is essential and the resulting charge distribution depends on the details of $\rho_{i}$. For instance, if one sets $\rho\left(q, q^{\prime}\right)$ to $\delta(q) \delta\left(q^{\prime}\right)$ the probabilities $\Pi_{f}(q)$ do not depend on $\phi$ :

$$
\Pi_{f}(q)=\sum_{N} \delta(q-N) \int d \phi P(N, \phi) .
$$

A similar result for a simple Josephson junction model was cited in [9]. A more general choice of $\rho_{i}$ preserves the $\phi$ dependence. One can summarize the situation by saying that $N$ and $\phi$ are related to canonically conjugated variables $q$ and $x$, which hampers their simultaneous measurement.

Since $\rho(x, p)$ are not positive in general, the $P(N, \phi)$ do not have to be positive. It might seem that these "negative probabilities" cannot be measured. Fortunately, it is not so. To understand this, let us see how one would measure $P(N)$ in the classical case. The only exact way is to make use of Eq. (16). One thus measures $\Pi_{i, f}$ separately and then obtains $P(N)$ from a deconvolution procedure: the Fourier transform of $P$ is the ratio of Fourier transforms of $\Pi$ 's. Our main result is that the same deconvolution procedure can be applied to Eq. (15), resulting in

$$
P(N, \phi)=\int \frac{d \chi}{2 \pi} e^{i N \chi} \frac{\rho_{f}(\phi+\chi / 2, \phi-\chi / 2)}{\rho_{i}(\phi+\chi / 2, \phi-\chi / 2)} .
$$

Since off-diagonal entries of the density matrix cannot be measured, this expression is not directly applicable. In [14] a scheme was proposed, showing how this can be circumvented by a repeated measurement of differently prepared initial density matrices. This allows one to characterize and measure $P(N, \phi)$, whatever sign it has.
In conclusion, we have studied the statistical properties of supercurrent in short constrictions. An extension of the Keldysh technique to account for full counting statistics of systems containing superconductors was developed. In the case of the supercurrent through a short constriction (point contact or tunnel junction) the cumulant generating function can be found quite generally. It shows that charge transfer occurs in units of $e$ and $2 e$, which largely enhanced probabilities in the case of contacts with large transmission. The charge transfer occurs in long trains of electrons passing through the contact in either direction, which is a signature of the coherent nature of the supercurrent. The relative probability of trains in the two directions is determined by the thermal occupation and the switching rate between them by the broadening parameter in the bulk of the superconductors. For tunnel junctions or point contacts between gapless superconductors we find the occurrence of negative values of $P(N, \phi)$, which questions the interpretation as a probability. Accounting for the full time evolution of an (idealized) measuring device, we have shown that these negative values can indeed be observed.

We acknowledge useful discussions with L. S. Levitov and C. W. J. Beenakker. W. B. was financially supported by the "Stichting voor Fundamenteel Onderzoek der Materie" (FOM) and the "Alexander von Humboldt-Stiftung."

*Present address: Department of Physics and Astronomy, Klingelbergstrasse 82, 4056 Basel, Switzerland.

[1] A. Shnirman et al., Phys. Rev. Lett. 79, 2371 (1997); J. E. Mooij et al., Science 285, 1036 (1999); L. B. Ioffe et al., Nature (London) 398, 679 (1999).

[2] T. Hoss et al., Phys. Rev. B 62, 4079 (2000).

[3] J. C. Cuevas et al., Phys. Rev. Lett. 82, 4086 (1999); Y. Naveh and D. V. Averin, ibid. 82, 4090 (1999).

[4] A. Martín-Rodero et al., Phys. Rev. B 53, R8891 (1996).

[5] D. Averin and H. T. Imam, Phys. Rev. Lett. 76, 3814 (1996).

[6] L. S. Levitov et al., J. Math. Phys. (N.Y.) 37, 4845 (1996).

[7] J. Rammer and H. Smith, Rev. Mod. Phys. 58, 323 (1986).

[8] Yu. V. Nazarov, Ann. Phys. (Leipzig) 8, Special Issue, SI-193 (1999).

[9] G. Schön and A. D. Zaikin, Phys. Rep. 198, 237 (1990).

[10] G. Eilenberger, Z. Phys. 214, 195 (1968); A. I. Larkin and Yu. N. Ovchinnikov, Sov. Phys. JETP 26, 1200 (1968); K. D. Usadel, Phys. Rev. Lett. 25, 507 (1970).

[11] Yu. V. Nazarov, Superlattices Microstruct. 25, 1221 (1999).

[12] B. A. Muzykantskii and D. E. Khmelnitskii, Phys. Rev. B 50, 3982 (1994).

[13] It is interesting to note that in the limit $\delta \rightarrow 0$ the action has poles for energies $E_{B}^{2}(\chi)=\Delta^{2}\left[1-T \sin ^{2}\left(\frac{\phi \pm \chi}{2}\right)\right]$. The counting field therefore couples directly to the phase sensitivity of the Andreev bound states.

[14] Yu. V. Nazarov and M. Kindermann, cond-mat/0107133 (unpublished). 